\title{
A TECHNIQUE FOR MEASURING THE RELATIVE CESR BEAM ENERGY*
}

\begin{abstract}
$\underline{\text { S. Henderson }}^{\dagger}$, V. Boisvert, K. Finkelstein, D. Abstract

The beam energy at the Cornell Electron Storage Ring must be maintained within $1 \mathrm{MeV}$ of the $\Upsilon(4 S)$ resonance peak. The beam energy is determined on a run-by-run basis by calculation based upon machine parameters. As a test of the energy calculation we have explored a technique for determining the relative CESR beam energy using synchrotron radiation. We describe the technique and present a status report on our experimental investigations.
\end{abstract}

\section{INTRODUCTION}

The Cornell Electron Storage Ring (CESR) operates on and just below the $\Upsilon(4 S)$ resonance $(\sqrt{s}=10.58 \mathrm{GeV})$ providing $e^{+} e^{-}$collisions for the CLEO experimental detector. The resonance peak is traditionally found by performing lengthy beam energy scans. Following such a scan the CESR beam energy is determined on a run-by-run basis by calculation based upon the machine parameters (RF frequency, measured dipole field, corrector strengths, electrostatic separator voltages, etc.) while taking into account the magnetic history. For maintenance of the beam energy over the course of many months we rely on the energy program to properly predict the relative energy changes. In order to maximize the physics event rate and to simplify data analysis, it is necessary to maintain the CESR beam energy within $1 \mathrm{MeV}$ of the resonance peak (the $\Upsilon(4 S)$ resonance width is $10 \mathrm{MeV}$ ).

We seek a method for verifying the beam energy calculation under actual operating machine conditions which include pretzel orbits, detector solenoid and compensation, and a rippled vertical orbit. If such a method were realizable and capable of functioning at full CESR beam currents, we would envisage incorporation of the measurement technique into routine operations, although our primary motivation at present is to provide verification for the energy calculation. Thus we seek a non-invasive, rapid measurement technique with relative precision of $\sim 2 \times 10^{-4}$.

The traditional method of beam energy measurement at electron storage rings is that of resonant depolarization [1], which provides quite accurate energy calibration with relative precision of $\sim 10^{-5}$. Resonant depolarization was used at CESR to determine the $\Upsilon(1 S)$ mass [2]. Another class of measurement techniques which have been proposed utilize synchrotron radiation (SR) in one way or another. A solid-state detector was used at BESSY to measure the SR spectrum directly for determination of the critical energy [3]. Several proposals based on synchrotron radiation have

\footnotetext{
* Work supported by the National Science Foundation.

† Email: sdh9@ cornell.edu
}

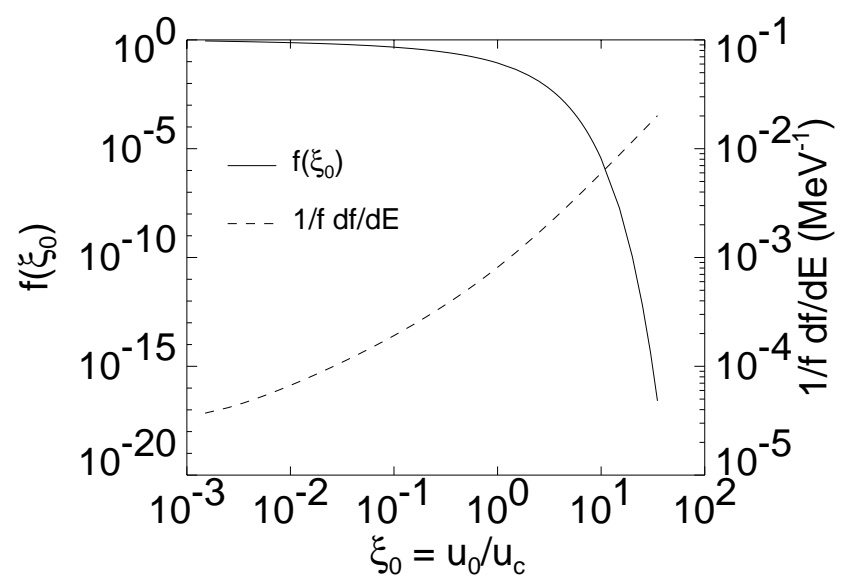

Figure 1: Integrated synchrotron radiation spectrum, $f$, and the sensitivity, $1 / f d f / d E$ as functions of the cutoff energy $\xi_{0}$. The sensitivity assumes $E=5.290 \mathrm{GeV}$.

been described for measurement of the TJNAF electron beam energy [4]. We describe a technique for measuring relative beam energy changes based on synchrotron radiation and present our preliminary findings.

\section{METHOD}

The method that we present exploits the very steep energy dependence of the SR spectrum at $x$-ray energies, $u$, several times the critical energy, $u_{c}$. Define the fraction of SR photons above a given energy $u_{0}=\xi_{0} u_{c}$ as

$$
f\left(\xi_{0}\right)=\int_{\xi_{0}}^{\infty} \frac{d \bar{N}}{d \xi} d \xi
$$

where $d \bar{N} / d \xi$ is the normalized SR energy spectrum and $\xi=u / u_{c}$. Because of the steep energy dependence of the SR spectrum, the integrated SR flux $f\left(\xi_{0}\right)$ is quite sensitive to the cutoff energy, $u_{0}$, for $u_{0} \gg u_{c}$ as shown in Figure 1.

The fractional change in SR flux above the cutoff energy for a given change in energy $\Delta \xi_{0}$ is

$$
\frac{1}{f} \frac{d f}{d \xi_{0}} \Delta \xi_{0}=-\frac{1}{f} \frac{d \bar{N}}{d \xi}\left(\xi_{0}\right) \Delta \xi_{0},
$$

or in terms of the beam energy $E$,

$$
\frac{1}{f} \frac{d f}{d E}=\frac{1}{f} \frac{d f}{d \xi_{0}} \frac{d \xi_{0}}{d E}=\frac{3 \xi_{0}}{E} \frac{1}{f} \frac{d \bar{N}}{d \xi}\left(\xi_{0}\right)
$$

If the integrated SR flux above a given energy $u_{0}$ could be measured, then for a beam energy change $\Delta E$, the fractional change in integrated flux is $1 / f d f / d E \Delta E$. This 
quantity is displayed in Figure 1, and shows the sensitivity of such a beam energy measurement technique as a function of cutoff energy $\xi_{0}$. For example, at $u_{0}=10 u_{c}$, a $1 \mathrm{MeV}$ change in beam energy changes the integrated flux by $0.57 \%$. Clearly, if one could monitor the SR flux above $\sim 10 u_{c}$ with accuracy $0.1 \%$, then one could measure relative beam energy changes rather accurately.

As a way of "integrating" the SR flux above a given energy, we use the $K$-edge of a high- $Z$ material selected so that the edge energy $E_{K} \gg u_{c} . x$-rays with energy above the $K$-edge are (primarily) photoabsorbed, and a fraction of those $x$-rays produce characteristic $K$-shell $x$-ray fluorescence emission. The flux of characteristic $x$-ray emission measured with an $x$-ray detector is proportional to the SR flux above the $K$ edge, which depends on the beam energy. To be precise, the rate of $K$-shell emission is given by the integral of the SR spectrum folded with the energy dependent photoabsorption cross-section which at falls like $u^{-7 / 2}$; this varies more slowly than the SR spectrum which at high energies falls like $e^{-\xi} / \sqrt{\xi}$. As a result, the observed sensitivity will be somewhat different from that shown in Figure 1.

Since the $K$-shell emission counting rate is beam current dependent and also depends on details of the SR source, target and detector geometry, a normalization is required. In addition to $K$-shell emission, the target also emits $L$-shell characteristic $x$-rays from photoabsorption of SR photons having energies between the $L$ and $K$ edges. The $L$-shell emission makes an ideal normalization since it is measured simultaneously with the $K$-shell emission in the same detector. Problems associated with combining measurements from two detectors having different stability and gains are avoided. The $L$-shell emission will also depend on beam energy, but since typically $E_{L} \simeq 1 / 6 E_{K}$, the dependence is much smaller than the $K$ emission dependence, as shown in Figure 1.

\section{EXPERIMENTAL SETUP}

The measurements reported in this paper were performed at the Cornell High Energy Synchrotron Source (CHESS) facility at one of the dipole bending magnet stations. The experimental layout is shown in Figure 2. A white SR beam of critical energy $10.4 \mathrm{keV}$ from a bending magnet ( $\rho=31.7 \mathrm{~m}$ ) was collimated horizontally with a $\mathrm{Pb}$ mask and filtered with a 0.25 in Al plate. The Al filter hardens the SR spectrum in order to reduce the flux of $L x$-rays to tolerable levels. All adjustable vertical slits were retracted to avoid intercepting the beam which would modify the spectral distribution at low energies. The SR beam is then brought into the experimental station where the intensity is monitored with an ion chamber. The beam strikes a $125 \mu \mathrm{m}$ gold foil placed $45^{\circ}$ to the incident beam. The SR source to target distance is $13.5 \mathrm{~m}$. The gold $K$ edge is 80.7 $\mathrm{keV}\left(7.8 u_{c}\right)$ and the $L$ edges are $\sim 13 \mathrm{keV}\left(1.3 u_{c}\right)$. The foil has attenuation factors $\mu t$ of 2.9 at the $K$ edge and 61 at the $L$ edge. At $90^{\circ}$ to the beam direction a Ge solid-state

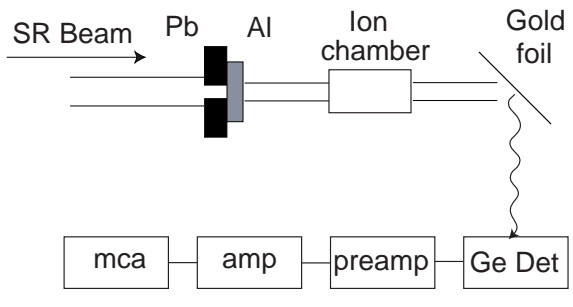

Figure 2: Experimental Layout

detector views the gold foil. Standard $x$-ray spectroscopy instrumentation is used to amplify and shape the pulse and the signal is then digitized in a multi-channel analyzer.

Figure 3 shows a typical $x$-ray spectrum obtained with this setup. The lines near $\sim 70 \mathrm{keV}$ are the characteristic $K$ shell emission and the lines near $\sim 10 \mathrm{keV}$ are the $L$-shell lines. The energy resolution of the system is $0.3 \mathrm{keV}$ at 69 $\mathrm{keV}$ (Au $K \alpha_{1}$ ). Generally, the beam current was limited to less than $0.5 \mathrm{~mA}$ so that the detector counting rate remained below $\sim 5 \mathrm{kHz}$ in order to minimize pulse pileup. As is evident, the spectrum is remarkably clean; $K$ and $L$ lines are easily identified and virtually free of background from other scattering sources.

We define a $K$ to $L$ counting rate ratio as follows:

$$
R_{K / L}=\frac{N_{K \alpha}-B_{K \alpha}+N_{K \beta}-B_{K \beta}}{N_{L}-B_{L}}
$$

where $N_{K \alpha}$ is the sum of counts in both $K \alpha$ peaks, $N_{K \beta}$ is the sum of counts in both $K \beta$ peaks, $N_{L}$ is the sum of counts in all $L$ peaks, and the $B$ 's are background count rates contained in separate background subtraction windows.

\section{RESULTS AND DISCUSSION}

To model the sensitivity of this method, an $x$-ray scattering Monte-Carlo simulation which includes Compton scattering, Rayleigh scattering, photoabsorption and $K$ and

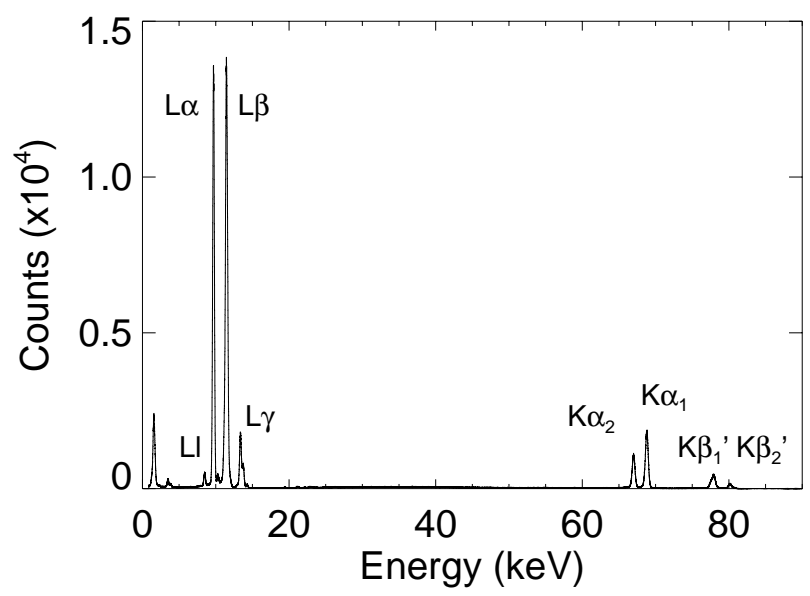

Figure 3: A typical $x$-ray spectrum from the gold foil. 
$L$ shell emission was employed. This simulation predicts that $R_{K / L}$ increases with beam energy at the rate of $0.21 \% / \mathrm{MeV}$ (for $E \simeq 5290 \mathrm{MeV}$ ) for the basic experimental conditions described above. We therefore require a combined statistical and systematic precision in $R_{K / L}$ of better than $0.2 \%$. Since we need a technique which will reliably measure relative energy changes regardless of how they arise, we need to verify that this method is insensitive to changes in SR source position, angle and size. An obvious concern is the source magnet field uniformity. The CESR dipoles have uniformity $\sim 10^{-4}$ over $\pm 2 \mathrm{~cm}$ about the center, resulting in a beam energy error of only $\sim 0.25$ $\mathrm{MeV}$ over the full range. A more significant effect is clipping of the SR beam on apertures in the $x$-ray beamline which has the potential to modify the spectral distribution. Other concerns arising from changes in SR source angle include the thickness uniformity of the gold target and $\mathrm{Al}$ filter. In our efforts to date, we have tested the viability of this technique by exploring several of these factors.

Figure 4 shows measured ratios $R_{K / L}$ (taken during a single machine studies period) plotted versus run time, where individual runs correspond to various changes in machine conditions. The data were obtained by collecting spectra for 8 minutes per point. The error bars are due to counting statistics. The first three data points were taken under identical conditions, and constitute the "reference" conditions. The remaining points show the effect of changes relative to these reference conditions. The next two data points show the effect of a vertical angle bump at the source $( \pm 40 \mu \mathrm{rad})$ corresponding to $\pm 0.4 \mathrm{~mm}$ motion on the target. We conclude that we were not "clipping" on vertical apertures in the $x$-ray beamline. For the next point the electrostatic horizontal separators were turned off, changing the horizontal angle at the SR source by $\sim 2 \mathrm{mrad}$. The next two points correspond to the reference conditions but with smaller and larger beam currents. In the following run, the vertical beamsize was increased by more than a factor of two. Despite rather significant changes in source angle, size and beam current, the ratio $R_{K L}$ remains constant within statistical errors, except perhaps for the latter data point.

Finally the CESR dipole field was increased by $1 \mathrm{G}$ (from nominal $B=2010 \mathrm{G}$ ), and the measured ratio increases by $0.97 \pm 0.19 \%$. By changing the dipole field, the beam energy is increased by the factor $\Delta B / B$ (giving $\Delta E=2.6 \mathrm{MeV}$ ), but the field at the source magnet is also increased so that the SR critical energy is increased by $\Delta u_{c}=3 u_{c} \Delta B / B$ (whereas for a beam energy change alone, $\Delta u_{c}=2 u_{c} \Delta E / E$ ). The measured value is in good agreement with the simulation prediction of $0.83 \%$.

The results of another dipole field dependence measurement are shown in Figure 5, along with the slope predicted by the simulation. Again, the data show good agreement with expectations.

In other machine studies sessions, the data were not always as reproducible as shown in Figure 4. It is thought that this may be due to clipping of the $x$-ray beam on ver-

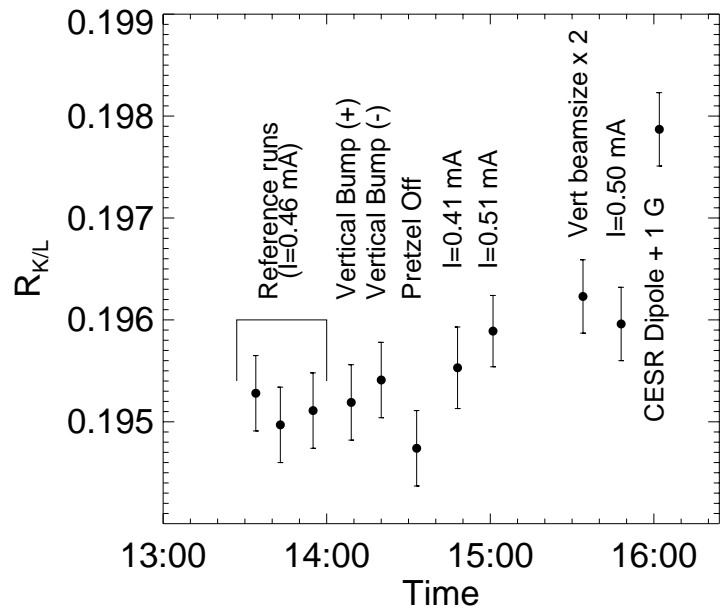

Figure 4: Measured $R_{K / L}$ for various machine conditions.

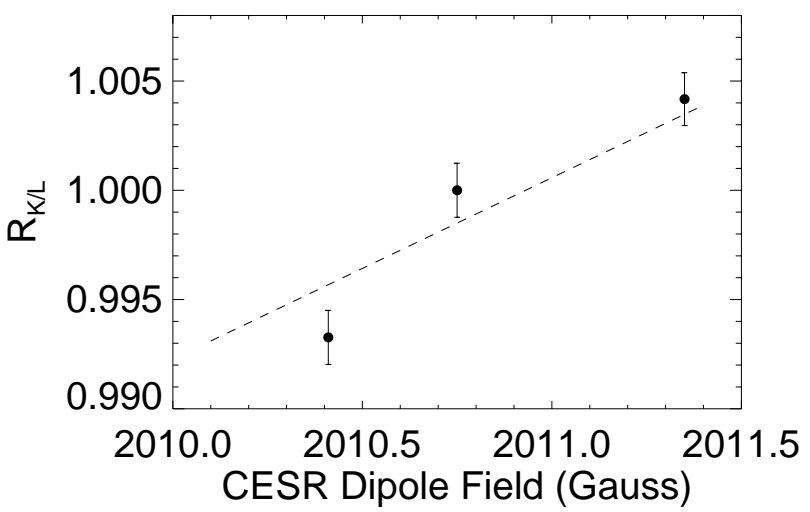

Figure 5: Measured $R_{K / L}$ (normalized to the middle point) versus CESR dipole field. The slope predicted by the simulation is overlayed.

tical apertures which would affect the spectrum, although this effect is still under investigation.

\section{SUMMARY}

The SR-based method that we describe for measuring relative beam energy changes shows promise. We are able to measure energy changes consistent with expectations. Our efforts in the near future will center on understanding the various factors that limit the reproducibility of the measurements and on increasing the count rate capability.

\section{REFERENCES}

[1] A.A. Sokolov and I.M. Ternov, Sov. Phys. Dokl. 8, 1203 (1964).

[2] W. W. MacKay et. al., Phys. Rev. D29 (1984) 2483.

[3] E. Tegeler and G. Ulm, NIM A266 (1988) 185.

[4] I.P. Karabekov, D.L. Egikian and C. Yang, NIM A286 (1990) 37; I.P. Karabekov and R. Rossmanith, NIM A321 (1992) 18; I.P. Karabekov and George R. Neil, NIM A356 (1995) 181. 